\title{
A Comparison of the Query Execution Algorithms in Secure Database System
}

\author{
Young-Dal Jang, Ji-Hong Kim \\ Department ofInformation and Telecommunication, SemyungUniversity, Korea
}

\begin{tabular}{l} 
Article Info \\
\hline Article history: \\
Received Jul 28, 2015 \\
Revised Nov 21, 2015 \\
Accepted Dec 2, 2015 \\
\hline Keyword: \\
Bloom Filter \\
Bucket Index \\
DAS (database as a service) \\
Database encryption
\end{tabular}

\begin{abstract}
In accordance with the database management, DAS (database as service) model is one solution for outsourcing. However, we need some data protection mechanisms in order to maintain the database security Themost effective algorithm to secure databases from the security threat of third party attackers is to encrypt the sensitive data within the database. However, once we encrypt the sensitive data, we have difficulties in queries execution on the encrypted database. In this paper, we focus on the search process on the encrypted database. We proposed the selective tuple encryption method using Bloom Filter which could tell us the existence of the data. Finally, we compare the search performance between the proposed method and the other encryption methods we know.
\end{abstract}

Copyright () 2016 Institute of Advanced Engineering and Science. All rights reserved.

\section{Corresponding Author:}

Ji-Hong Kim,

Departement of Information and Telecommuincation, Semyung University,

65, Semyeong-ro, Jecheon-si, Chungcheongbuk-do, Korea.

E-mail: jhkim@semyung.ac.kr

\section{INTRODUCTION}

The new trend of DAS system [1] leads to a new concern, security. Especially, when the sensitive information is contained in the database, it needs its confidentiality in such a model. In a typical setting of the problem, the confidential portions of the data should be stored at a remote location in an encrypted form at all times. For example, data encryption becomes important when the client chooses to hide away certain contents from server-side entities. Two new challenges emerge: (1) How to encrypt the sensitive data. (2) How to support queries on the encrypted relational data.

In order to query effectively on the encrypted database, user query should be translated to the modified form. The modified query should be suitable to search the data in the encrypted database stored in a DB server. Therefore we use the Bloom Filter and Bucket index for the index on the encrypted database search. The metadata in aclient module has hash functions for generating Bloom Filter and the bucket index for the numerical attributes. Therefore the original query is converted to the modified query with the help of the metadata. The index in server part is used to search the exact data from the encrypted database.

The remainder of this paper is organized as follows. Section 2 describes the general database encryption methods. Section 3 shows the details of the proposed method and Section 4 shows the comparison between the proposed method and the other methods. Finally, we conclude with a summary and directions for future work.

\section{RELATED WORK}

In general, we can classify the database encryption methods according to the encryption unit. There arethe element based encryption methods, the column/row based encryption methods, and the table based encryption methods. 
Table 1. The comparison between various Encryption Methods

\begin{tabular}{lllll}
\hline Encryption Unit & Encryption Speed & Memory Size & \multicolumn{2}{c}{ Search Speed } \\
& & & Keyword Search & Numeric Operation \\
\hline Element & Slow & Large & High & Low \\
Column/Row & Medium & Medium & Medium & Medium \\
Table & Fast & Small & Low & Low \\
\hline
\end{tabular}

The element based encryption method is useful only for the search for the same valued data, but it is not good for the numeric operations. It always needs to do the element level decryption before doing numeric operation. For example, there is one plain text table such as $\boldsymbol{R}\left(\boldsymbol{A}_{1}, \boldsymbol{A}_{2}, \boldsymbol{A}_{3}, \ldots, \boldsymbol{A}_{\boldsymbol{n}}\right)$. In this case, we store an encrypted relation $R^{e}\left(A_{1}^{e}, A_{2}^{e}, A_{3}^{e}, \ldots, A_{n}^{e}\right)$ where each attribute $\boldsymbol{A}_{i}^{\boldsymbol{e}}$ corresponds to the encrypted data for each attribute $\boldsymbol{A}_{\boldsymbol{i}}$.

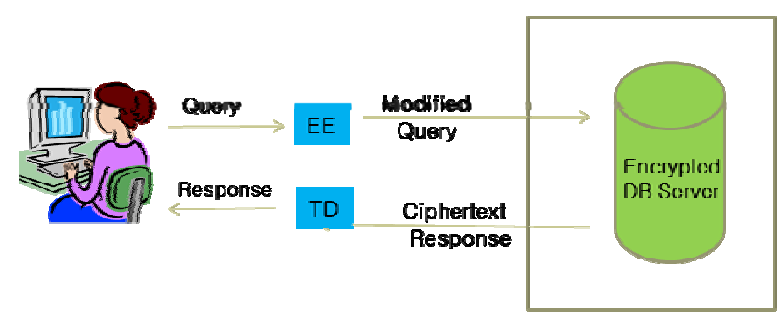

Figure 1. The element based encryption method

Table 2. The original query and the modified query

\begin{tabular}{cc}
\hline The original plain-text query & The transformed query \\
\hline SELECT name & SELECT name \\
FROM std_info & FROM std_info(E) \\
WHERE city $=$ seoul & WHERE city = seoul(E) \\
\hline
\end{tabular}

In Figure1, a query generated by a database user is transformed to the modified query which could search the exact data from the encrypted database. In Table 2, if the std info table is the student personal information, std_info(E) is the encrypted database which contains the encrypted elements in the std_info table. The "seoul(E)" is the encrypted form of the seoul

The column/row based encryption method is the method which encrypts the data by the unit of the column or row. Typically, the row based encryption method which is also called by tuple based encryption method, is generally used.

For each relation $R\left(A_{1}, A_{2}, A_{3}, \ldots, A_{n}\right)$, we store on the server an encrypted relation $R^{e}$ (etuple, $\left.A_{1}^{b i}, A_{2}^{b i}, A_{3}^{b i}, \ldots, A_{n}^{b i}\right)$ where the etuple stores an encrypted string that corresponds to a tuple in relation $\mathrm{R}$. Each attribute $A_{i}^{b i}$ corresponds to the bucket index for the attribute $A_{i}$ that will be used for query processing at the server. In this case we have to map the domain of values of attribute $R . A_{i}$ into partition $\left\{p_{1}, p_{2}, p_{3}, \ldots\right.$, $p k$ such that these partitions taken together cover the whole domain; and any two partitions do not overlap. Formally, we define a function partition as follows; partition $\left(R . A_{i}\right)=\left\{p_{1}, p_{2}, p_{3}, \ldots, p_{k}\right\}$. We will call k the number of the bucket. This method is proposed by Hore [2] and known to be a generally good method to protect the database these days. 


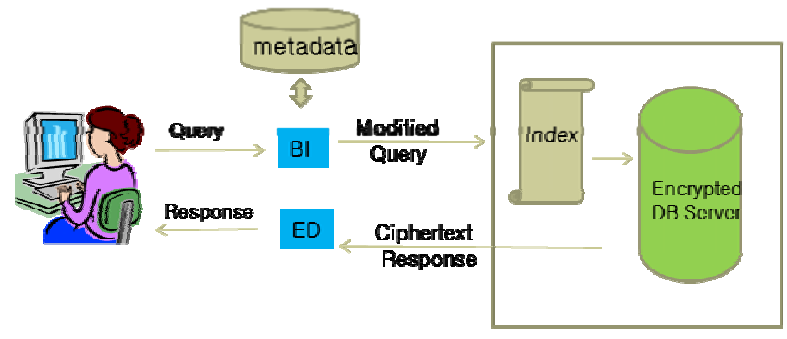

Figure 2. The typical tuple encryption method

Table 3 . The original query and the modified query

\begin{tabular}{cc}
\hline The original plain-text query & The transformed query \\
\hline SELECT name & SELECT etuple \\
FROM std_info & FROM std info(E) \\
WHERE age $=30$ & ${\text { WHERE }(\text { age })^{\mathrm{bi}}=\text { age }(30)^{\mathrm{bi}}}$
\end{tabular}

In Figure 2, BI means the Bucket Index used for query conversion process and ED means etuple decryption process. A query originated from a user is transformed to the modified query in order to search the exact data from the encrypted database. The metadata store the bucket index of the each attribute. So, the value of the attribute involved in the user query is converted to the bucket index. Then the bucket index value is inserted into the modified query which could search the exact data from the tuple encrypted database. The query result returned from the server is the encrypted string etuple. The client module should decrypt etuple and extract the right result from the decrypted data.

In Table 3, the std_info table contains the plaintext form of the student personal information and thestd_info(E) is the encrypted database which contains the encrypted string of each tuple. The $a g e^{b i}$ is the bucket index value of the age element.

Finally, table based encryption method, is used to make the backup data in order to store data on the original database periodically. Therefore, this method is not good for query processing on the encrypted database.

\section{THE PROPOSED DATABASE ENCRYPTION MODEL}

Even if some sensitive data exists in the database, there is still a lot of insensitive data in the database. So we proposed the selective tuple encryption method with Bloom Filter. A Bloom Filter [3] is a simple space-efficient randomized data structure used to represent the existence of the data. The filter is a bit array over which membership queries are conducted to distinguish between members of the given set or not. Hash function techniques are used to both save space and allow member lookup. Bloom Filters allow false positives but the space savings often outweigh this drawback when the probability of an error is controlled. The Bloom Filter is an array of $\mathrm{m}$ bits, initially all set to 0 . The Bloom Filter uses $\mathrm{k}$ independent hash functions with range $\mathrm{m}$. The number of the sample space is $\mathrm{n}$ elements. After inserting $\mathrm{n}$ elements with $\mathrm{k}$ hash functions in the $\mathrm{m}$ bits Bloom Filter, the probability of the false positive error [3] is $\mathrm{f}=(1-(1-$ $1 \mathrm{mkn} \mathrm{k} \approx 1-\mathrm{e}-\mathrm{knmk}$. This false positive rate could be reduced by choosing the large number of $\mathrm{m}$ in proportion to the number of input element $\mathrm{n}$. In Figure 3, The BF means the query conversion process using Bloom Filter value and ED means the selective etuple decryption process. The metadata store the bucket index of the each attribute. 


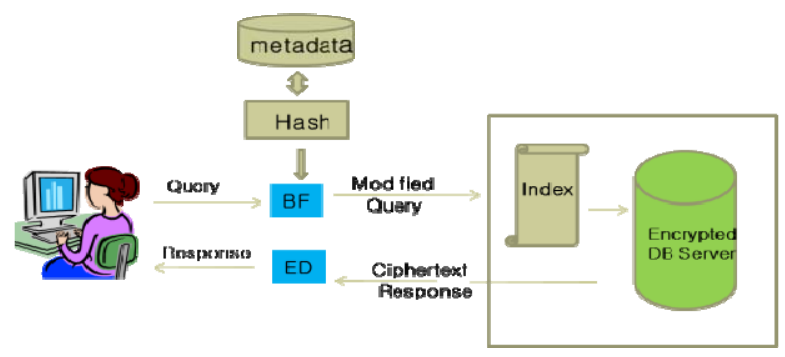

Figure 3. Selective tuple encryption with Bloom Filter method

We propose two kinds of encryption methods for the sensitive data only. The first type is the characteristic data involved in each attribute. The keyword for the search process is extracted from the each characteristic attribute. The city name "seoul" is one example as a keyword. The extracted keyword is hashed and the resulting value of the hash function is considered a bit number and the corresponding bits are set by one in the Bloom Filter, $\mathrm{BF}=\mathrm{Hash}(\mathrm{seoul})$. The second type is the numerical data involved in each attribute. The numerical data is converted to the bucket index which is stored in the metadata. The grade is one example as a numerical data. The value of the gradewould be divided by the large number of the buckets because the bucket index value is not disclosed, and only involved in the Bloom Filter using hash functions. So, the numerical attribute involved in the user query is converted to the bucket index first, then the bucket index value is converted to the corresponding bits of the Bloom Filter, according to the result of the hash functions.

A query originated from a user is transformed to the modified query in order to search the exact data from the encrypted database. We call this method SEBF (Selective Tuple Encryption with Bloom Filter). Table4 shows the query conversion process in SEBF. The generation process of the encrypted database in SEBF is shown in Figure 4.

Table 4. The query used in SEBF method

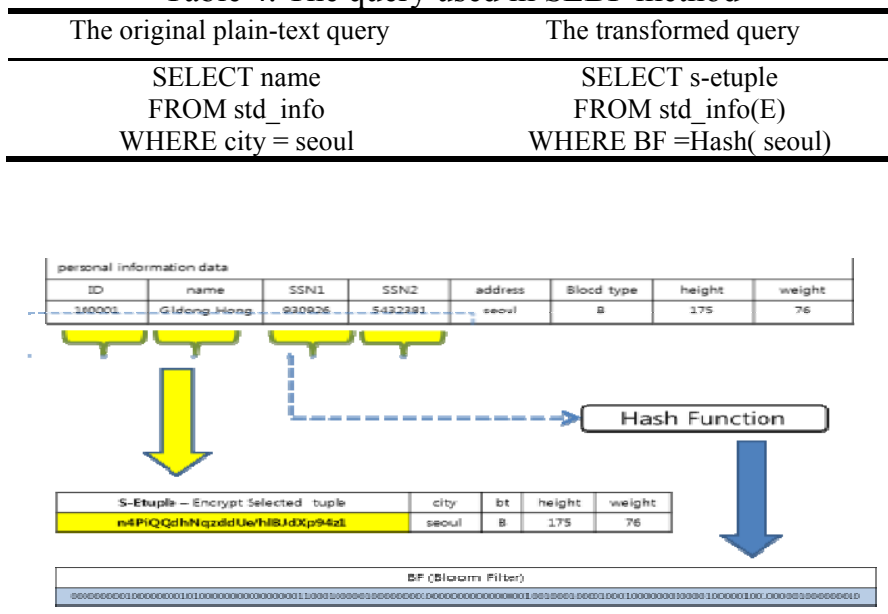

Figure 4. The structure of the SEBF method

\section{DISCUSSION AND CONCLSIONS}

In this chapter, we will compare the proposed encryption method with the other encryption methods.The First encryption method is the Tuple Encryption with Bucket Indexing method. We call this method as TEBI. This method is proposed by Hore $[2,5,6]$ and the same to the typical tuple encryption method. In Figure5, you can see that it only uses the bucket index regardless of the attribute data type. All of the tuple elements are encrypted by the e-tuple (encrypted tuple) and each attribute of the tuple is converted by bucket index. Therefore, we can search the data using the bucket index which is stored in the metadata. The e-tuple data is extracted from the database according to the bucket index. If we want to search the city name "seoul", then we first convert the city name to the according bucket index using the metadata in a client module. The modified query is sent to the database server, and query results, which are the e-tuples according 
to the bucket index, are returned from the server. The client should decrypt the corresponding e-tuples and could get the exact data. The fatal drawback of this method is the exposure of the bucket index value of the attribute. The attacker can guess the distribution of the data from the encrypted database by the value of the bucket index.

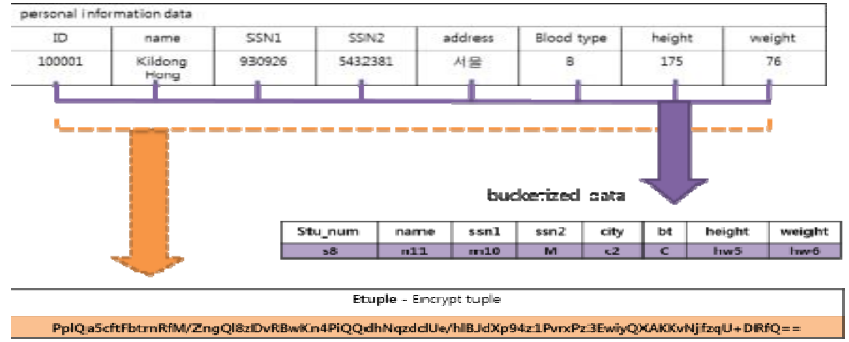

Figure 5. The structure of the TEBI method

Another method is the Tuple Encryption with Bloom Filter method [4, 7]. We call this method TEBF. In Figure 6, you can see that it uses the Bloom Filter with the e-tuple. In this method, the bucket index is replaced by the Bloom Filter. After each attribute of the tuple is converted to a bucket index, the bucket index value is converted to the corresponding bits of the Bloom Filter according to the result of the hash functions. A query originated from a user is transformed to the modified query in order to search the exact data from the encrypted database.

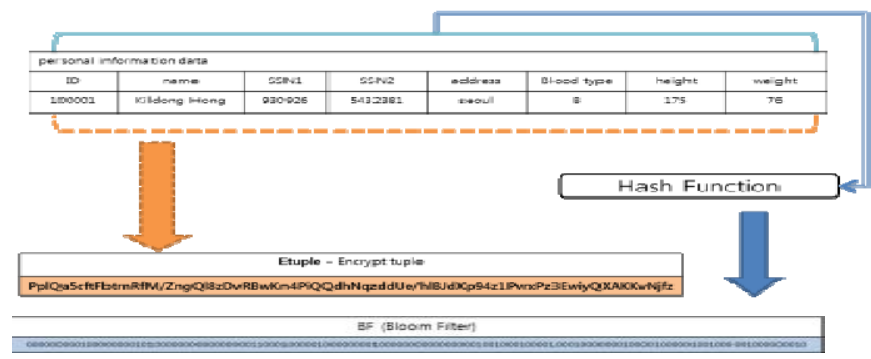

Figure 6. The structure of the TEBF method

This method is more secure than the TEBI method previously mentioned, but all the elements are encrypted regardless of the sensitivity of the data. Even if we want to search the one or two attributes, we have to decrypt all of the according e-tuples. Now, we compare the search performance between the plaintext and the three encryption methods (TEBI, TEBF, SEBF). The SEBF is the proposed method in this paper. In order to compare three database encryption methods, TEBI and TEBF use the bucket index rather than keyword searceh. Only SEBF uses the keyword for searching the character type data and the bucket index for searching the numerical type data and the number of the bucket used in SEBF is about five times as many as TEBI and TEBF methods. It means that we divide the numerical attributes into more partitions in SEBF. In order to check the search performance of the three encryption methods, we use the following tables. std info(std_id, name, ssn1, ssn2,city, bt, height, weight), std grade(std id, grade, d id, average, e-grade, adviser) In TEBI, std_info ${ }^{e}$ and std_grade ${ }^{e}$ are composed of the e-tuple and bucket index of each attribute. In TEBF, std_info ${ }^{e}$ and std_grade ${ }^{e}$ are composed of the e-tuple and Bloom Filter value which is composed with the result of the hash functions using bucket index of each attribute. In SEBF, Std_grade table is not encrypted and some attributes (std id, name, ssn1 and ssn2) in the Std infotableare encrypted. Table 5 shows the two queries used for performance analysis. Table 6 shows the result of the performance test. Table 7 shows the performance comparison of each encryption method. 
Table 5. Two queies used for performance analysis

\begin{tabular}{|c|c|c|c|c|}
\hline \multicolumn{3}{|c|}{ Query1 } & \multicolumn{2}{|c|}{ Query2 } \\
\hline \multicolumn{3}{|c|}{$\begin{array}{c}\left.\text { SELECT count }{ }^{*}\right) \\
\text { FROM std_info, std_garde } \\
\text { WHERE (e-grade }>750) \text { AND } \\
\text { (city =jecheon) }\end{array}$} & \multicolumn{2}{|c|}{$\begin{array}{l}\text { FROM std_info, std_garde } \\
\text { WHERE (d-id=electronics)AND } \\
\text { (grade }>\text { average(grade) })\end{array}$} \\
\hline \multicolumn{5}{|c|}{ Table 6. The result of the search performance test } \\
\hline & $\begin{array}{c}\text { Return No } \\
\text { (Q1) }\end{array}$ & $\begin{array}{c}\text { Search } \\
\text { Time(Q1) }\end{array}$ & $\begin{array}{c}\text { Return No } \\
\text { (Q2) }\end{array}$ & $\begin{array}{c}\text { Search } \\
\text { Time(Q2) }\end{array}$ \\
\hline Plaintext & 764 & 0.0450026 & 2565 & 0.117067 \\
\hline TEBI & 7388 & 0.2150123 & 19981 & 0.4550263 \\
\hline TEBF & 7436 & 0.2480136 & 21996 & 0.618254 \\
\hline SEBF & 807 & 0.0650031 & 10018 & 0.310177 \\
\hline
\end{tabular}

Table 7. The comparison between three methods

\begin{tabular}{ccccccc}
\hline & $\begin{array}{c}\text { Encryption } \\
\text { Unit }\end{array}$ & Index & $\begin{array}{c}\text { Keyword } \\
\text { Search }\end{array}$ & $\begin{array}{c}\text { Numerical } \\
\text { Operation }\end{array}$ & Security & Comment \\
\hline TEBI & Tuple & BI & Low & Medium & Low & Disclose the distribution of the data. \\
TEBF & Tuple & BI, BF & Medium & Medium & High & $\begin{array}{c}\text { High speed only if we use large } \\
\text { number of bucket index. } \\
\text { SEBF }\end{array}$ \\
& Selective tuple & BI, BF & High & High & Medium & $\begin{array}{c}\text { Only sensitive data should be } \\
\text { encrypted }\end{array}$ \\
\hline
\end{tabular}

We can see that the SEBF method has the best search performance. But it is a very difficult problem to decide how much data would be encrypted according to the sensitivity. In fact, the search performance is different depending on the queries types we choose.If we use character type cipher-text as a search condition, SEBF has better performance than other methods because it uses keyword search. In the case of the aggregation operation, SEBF has better performance than other methods only if it uses the large numbers of the bucket index.

\section{ACKNOWLEDGEMENTS}

This paper was supported by the Semyung University Research Grant of 2015.

\section{REFERENCES}

[1] S. Vimercati, and S. Foresti, "Privacy of Outsourced Data", Auerbach Publications (Taylor and Francis Group), 320: 174-187.

[2] B. Hore, S. Mehrotra, and H. Hacigũ.mô.s, "Managing and Querying Encrypted data”, Handbook of Data Security, pp 163-190, 2008.

[3] A. Broder and M. Mitzenmacher. Network applications of Bloom Filters: A survey. Internet Mathematics, 1(4):pp.485-509, 2004.

[4] J. Kim, T. Sahama, S. Kim, "A Performance test of Query Operation on Encrypted Database", LNCS 235, pp 801810,2013

[5] B. Hore, S. Mehrotra, G. Tsudik, "A privacy-preserving index for range queries", Proceedings of the Thirtieth international conference on Very large databases- Volume30. VLDB '04, VLDB Endowment pp 720-731, 2004.

[6] H. Hacigü.mö.s, B. Iyer, C. Li, and S.Mehrotra, "Executing SQL over encrypted data in the database service provider model", In Proc. of theACM SIGMOD, pp. 45-49, 2002

[7] D. Shin, T. Shahama, J. Kim and J. Kim, "The Scalability and the Strategy for EMR Database Encryption Techniques", JICCE, pp. 556-582, 2011. 


\section{BIOGRAPHIES OF AUTHORS}

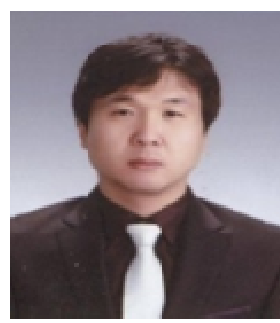

Young-Dal Jang (jang_yd@naver.com) received his B.S and M.S degrees in electronic engineering from Semyung University, Jecheon, Korea in 1998 and 2008.

$\mathrm{He}$ is currently in his Ph.D course in information and telecommunication in Semyung University, Jecheon, Korea.

His current research intersets include the netowrk securityand database security.

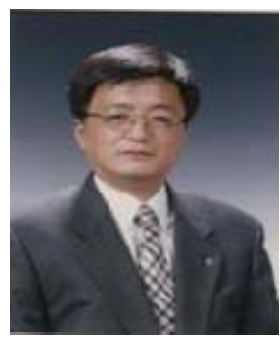

Ji-Hong Kim (jhkim@semyung.ac.kr) received his B.S degree in electronic engineering from Hanyang University, Seoul, Korea in 1982. and received his M.S and Ph.D degrees in electronic and communication engineering from Hanyang University, Seoul, Korea in 1984 and 1996.

He has worked in the Department of the information and telecommunication in Semyung University since 1991.

His current research intersets include the netowrk security and database security and cloud computing. 